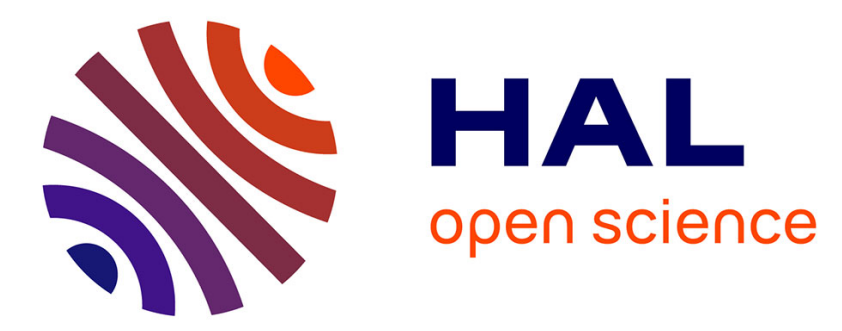

\title{
Multifunctional Amyloid Oligomeric Nanoparticles for Specific Cell Targeting and Drug Delivery
}

Weiqiang Wang, Rafayel Azizyan, Adriana Garro, Andrey Kajava, Salvador Ventura

\section{- To cite this version:}

Weiqiang Wang, Rafayel Azizyan, Adriana Garro, Andrey Kajava, Salvador Ventura. Multifunctional Amyloid Oligomeric Nanoparticles for Specific Cell Targeting and Drug Delivery. Biomacromolecules, 2020, 21 (10), pp.4302-4312. 10.1021/acs.biomac.0c01103 . hal-03431802

\section{HAL Id: hal-03431802 \\ https://hal.science/hal-03431802}

Submitted on 17 Nov 2021

HAL is a multi-disciplinary open access archive for the deposit and dissemination of scientific research documents, whether they are published or not. The documents may come from teaching and research institutions in France or abroad, or from public or private research centers.
L'archive ouverte pluridisciplinaire HAL, est destinée au dépôt et à la diffusion de documents scientifiques de niveau recherche, publiés ou non, émanant des établissements d'enseignement et de recherche français ou étrangers, des laboratoires publics ou privés. 


\title{
Multifunctional Amyloid Oligomeric Nanoparticles for Specific Cell 2Targeting and Drug Delivery
}

\author{
3 Weiqiang Wang, Rafayel A. Azizyan, Adriana Garro, Andrey V. Kajava, and Salvador Ventura*
}

Cite This: https://dx.doi.org/10.1021/acs.biomac.0c01103

Read Online

4 ABSTRACT: Natural selection has endorsed proteins with amazing structures 5 and functionalities that cannot be matched by synthetic means, explaining the 6 exponential interest in developing protein-based materials. Protein self-assembly 7 allows fabricating complex supramolecular structures from relatively simple 8 building blocks, a bottom-up strategy naturally employed by amyloid fibrils. 9 However, the design of amyloid-inspired materials with biological activity is 10 inherently difficult. Here, we exploit a modular procedure to generate functional 11 amyloid nanostructures with tight control of their mesoscopic properties. The soft 12 amyloid core of a yeast prion was fused to dihydrofolate reductase through flexible

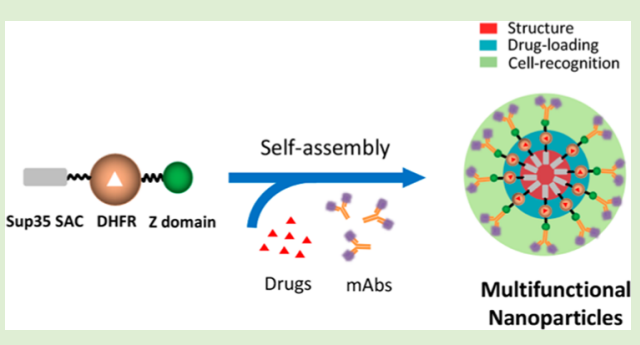

13 linkers of different sizes. This enabled us to produce, for the first time, biocompatible protein-only amyloid-like oligomeric 14 nanoparticles with defined dimensions in which the embedded enzyme remained highly active, as assessed by biophysical and 15 enzymatic assays. The modular design allowed one to obtain multifunctional nanoparticles by incorporating the antibody-binding Z16 domain to the protein fusion. We show how these assemblies can be exploited for antibody-directed targeting of specific cell types 17 and the localized delivery of methotrexate, resulting in the intracellular uptake of the drug by cancer cells and their death. Overall, 18 the novel protein particles we describe in this work might find applications in areas as diverse as biocatalysis, bioimaging, or targeted 19 therapies.

\section{INTRODUCTION}

21 The design and production of biologically inspired assemblies 22 that can be exploited for the fabrication of functional materials 23 is a rapidly growing area of research. ${ }^{1}$ Amyloid fibrils have been 24 traditionally associated with human diseases. ${ }^{2}$ However, the 25 recent discovery of amyloids assisting biological functions in a 26 wide range of organisms, from bacteria to humans, ${ }^{3}$ has 27 changed this perception and inspired the use of their unique 28 architecture to build up nanostructured materials for 29 applications in biomedicine and biotechnology. ${ }^{4}$

30 A majority of the available amyloid-based functional 31 nanomaterials result from the self-assembly of short synthetic 32 peptides of natural or artificial origin. ${ }^{5}$ In contrast, the 33 assembly of globular proteins into amyloid-like materials with 34 biological activity has been less successful. This is expected 35 since the aggregation of globular domains into amyloid 36 structures necessarily implies a process of conformational 37 conversion in which they lose their native fold and thus their 38 activity. ${ }^{6}$

39 The main advantage of protein-based materials is the nature 40 of globular proteins, which allows one to alter the material 41 functionality by genetic redesign to fit the intended application. 42 We have recently succeeded in designing highly ordered 43 amyloid-like nanofibrils containing properly folded and highly 44 active proteins using a modular strategy. The distinctive 45 feature of the approach consists in the use of a soft amyloid 46 core (SAC) as the assembling unit. SACs are short 47 amyloidogenic sequence stretches initially identified in the disordered and low-complexity domains of yeast prions. ${ }^{8}$ They 48 differ from the classical amyloid cores of pathogenic proteins in 49 that they are slightly longer and more polar., ${ }^{9,10}$ This results in 50 a weaker and more diffuse amyloid propensity but still 51 sufficient to nucleate the self-assembly reaction. The SAC is 52 fused through a Gly/Ser soluble and flexible linker to the 53 globular protein of interest. The resulting fusion proteins are 54 produced recombinantly at high yield and in a soluble manner; 55 still, their ordered aggregation can be induced under defined 56 conditions, rendering functional synthetic amyloid fibrils in 57 which the SAC forms the amyloid spine and the globular 58 domains hang from it in an active conformation, as 59 demonstrated for fluorescent proteins and enzymes. ${ }^{7}$

60

We use the SAC (residues 100-118) of the Sup35 yeast 61 prion (Sup35-SAC) as the default assembling module. Its small 62 size allows one to use a connecting linker of only eight residues 63 and obtain amyloid fibrils in which the fused protein is active 64 without major steric impediments and with a reduced entropy 65 penalization for the assembly reaction. This is in contrast with 66 natural amyloid proteins displaying a similar architecture, like 67

Received: July 21, 2020

Revised: September 1, 2020

Published: September 4, 2020 
68 the prion proteins Het-s, ${ }^{11}$ Sup35p, and Ure2p, ${ }^{12}$ human 69 Rip1/Rip3, ${ }^{13}$ or the TAR-DNA binding proteins, ${ }^{14}$ where the 70 connecting linker is significantly longer ( $>50$ residues).

71 Recently, using molecular and mesoscopic modeling, we 72 were able to establish a theoretical relationship between the 73 size of a globular domain and the length of the shortest linker 74 that still allows the formation of infinite amyloid fibrils when 75 fused to an amyloidogenic sequence. ${ }^{15}$ The model suggested 76 that shortening the linker below this size limit would result in 77 the formation of oligomers of defined size instead of the typical 78 fibrils. $^{16,17}$ This might provide a unique opportunity to 79 generate small protein-based nanoparticles decorated with $\grave{a}$ 80 la carte globular protein functionalities.

81 Here, we connected Sup35-SAC to the dihydrofolate 82 reductase (DHFR) enzyme ${ }^{18}$ (Sup35-DHFR) and demon83 strate that we can control the mesoscopic properties of the 84 resulting assemblies just by playing with the linker length, 85 obtaining either catalytic fibrils or catalytic oligomeric 86 nanoparticles. These nanoparticles are spherical assemblies of 87 an amyloid-like nature but devoid of any toxicity. They are 88 homogenous in size, stable, and more active than the 89 corresponding fibrils.

90 We explored the potential of these novel protein-based 91 nanoparticles to generate functionalized materials by creating a 92 tripartite fusion in which we incorporated the Z-domain, ${ }^{19}$ an 93 engineered analog of the B-domain of the Staphylococcus aureus 94 protein $\mathrm{A}^{20}$ to the C-terminus of the Sup35-DHFR protein. 95 The new fusion protein also assembles into spherical 96 oligomeric nanoparticles that can now be decorated with any 97 antibody of interest, thanks to the Z-domain's nanomolar 98 affinity for antibodies' Fc domains, thus generating multivalent 99 particles.

100 We show that antibody-loaded nanoparticles can be directed 101 specifically against cancer cells and demonstrate that they can 102 be used as nanocarriers for cell-specific delivery of methotrex103 ate (MTX). MTX acts as an antagonist of folic acid, which is 104 necessary for DNA synthesis and accordingly is used as a 105 chemotherapeutic agent for the treatment of cancer. ${ }^{21,22}$ 106 However, it displays a weak pharmacokinetic profile, and 107 significant off-target toxicity ${ }^{23-25}$ and MTX-based nano108 medicines have been designed to overcome these drawbacks. ${ }^{26}$ 109 In our nanoparticles, the Z-domain-bound antibody acts as a 110 cellular selector and the DHFR moiety as the MTX carrier. 111 Once released from its carrier, the drug is internalized, 112 displaying a potent and localized cytotoxic effect. The strategy 113 is simple and modular and can be adapted to target any cell 114 type of interest.

\section{MATERIALS AND METHODS}

116 Reagents and Enzymes. Reagents and enzymes were purchased 117 from Sigma-Aldrich (U.K.) unless otherwise stated. Carbon grids 118 (400 square mesh copper) were purchased from Micro to Nano (The 119 Netherlands), and the uranyl acetate solution was provided by the 120 microscopy service at Universitat Autònoma de Barcelona. Sup35121 SAC peptides were purchased from CASLO ApS (Scion Denmark 122 Technical University).

123 Expression and Purification of Proteins. The cDNA of Sup35124 5aa-DHFR, cloned in the plasmid pET28(a) with a His6 tag, was 125 acquired from GenScript. The constructs pET28(a)/Sup35-8aa126 DHFR, pET28(a)/wild-type DHFR (DHFR-wt), and pET28(a)/ 127 Sup35-5aa-DHFR-Z were obtained by mutagenesis on top of the 128 plasmid pET28(a)/Sup35-5aa-DHFR. Escherichia coli BL21 (DE3)129 competent cells were transformed with the corresponding plasmids. 130 Then, transformed cells were grown in a $10 \mathrm{~mL}$ lysogeny broth (LB) medium containing $50 \mu \mathrm{g} \cdot \mathrm{mL}^{-1}$ kanamycin, overnight at $37^{\circ} \mathrm{C}$ and 131 transferred into $1 \mathrm{~L}$ fresh $\mathrm{LB}$ media containing $50 \mu \mathrm{g} \cdot \mathrm{mL}^{-1} 132$ kanamycin. After reaching an $\mathrm{OD}_{600}$ of 0.6 , the culture was induced 133 with $0.4 \mathrm{mM}$ isopropyl $\beta$-D-1-thiogalactopyranoside (IPTG) and 134 grown at $20{ }^{\circ} \mathrm{C}$ for $16 \mathrm{~h}$. Cells were collected by centrifugation at 135 $5000 \mathrm{rpm}$ for $15 \mathrm{~min}$ at $4{ }^{\circ} \mathrm{C}$. The collected pellet was resuspended 136 into $20 \mathrm{~mL}$ of phosphate-buffered saline (PBS) pH 7.4 containing 20137 $\mathrm{mM}$ imidazole, $1 \mathrm{mg} \cdot \mathrm{mL}^{-1}$ lysozyme, and $1 \mathrm{mM}$ PMSF. The solution 138 was incubated on ice, followed by sonication for $20 \mathrm{~min}$. The 139 supernatant was collected by centrifugation at $15000 \mathrm{rpm}$ for $30 \mathrm{~min} 140$ at $4{ }^{\circ} \mathrm{C}$ and purified using a nickel-charged IMAC column, followed 141 by a gel filtration onto a HiLoad Superdex 75 prep-grade column (GE 142 Healthcare). The purified proteins were frozen with liquid nitrogen 143 and stored at $-80{ }^{\circ} \mathrm{C}$. Sodium dodecyl sulfate-polyacrylamide gel 144 electrophoresis (SDS-PAGE) confirmed the purity of the samples. 145 The concentrations of the proteins DHFR-wt, Sup35-5aa-DHFR, 146 Sup35-8aa-DHFR, and Sup35-5aa-DHFR-Z were determined by 147 ultraviolet (UV) absorption using $\varepsilon$ values of $33585,38055,148$ 38055 , and $39420 \mathrm{~L} \cdot \mathrm{mol}^{-1} \cdot \mathrm{cm}^{-1}$, respectively.

149

Conformational Characterization. Proteins were dissolved at a 150 final concentration of $20 \mu \mathrm{M}$ in $\mathrm{PBS} \mathrm{pH} 7.4$ buffer; then, samples 151 were filtered through a $0.22 \mu \mathrm{m}$ Millipore filter and immediately 152 analyzed. Far-UV circular dichroism (CD) spectra were recorded 153 from 260 to $200 \mathrm{~nm}$ at $1 \mathrm{~nm}$ bandwidth, with a response time of $1 \mathrm{~s} 154$ and a scan speed of $100 \mathrm{~nm} \cdot \mathrm{min}^{-1}$ in a Jasco-710 spectropolarimeter 155 (Jasco Corporation, Japan), thermostated at $25{ }^{\circ} \mathrm{C}$. Ten accumu- 156 lations were averaged for each spectrum.

Aggregation Assay. Proteins were prepared at $200 \mu \mathrm{M}$ for 158 Sup35-5aa-DHFR and Sup35-8aa-DHFR and at $600 \mu \mathrm{M}$ for Sup35- 159 5aa-DHFR-Z in PBS pH 7.4 and filtered through a $0.22 \mu \mathrm{m}$ filter. The 160 samples were incubated at $25^{\circ} \mathrm{C}$, with agitation for four days. DHFR- 161 wt was incubated at the same concentrations and conditions. 162

Amyloid Dye Binding. Thioflavin-T (Th-T) and Congo red 163 (CR) were used to monitor the formation of amyloid assemblies. For 164 the Th-T binding assay, incubated proteins were diluted to a final 165 concentration of $20 \mu \mathrm{M}$ in PBS pH 7.4, in the presence of $25 \mu \mathrm{M}$ Th- 166 T. Emission fluorescence was recorded in the $460-600 \mathrm{~nm}$ range, 167 using an excitation wavelength of $445 \mathrm{~nm}$ and an emission bandwidth 168 of $5 \mathrm{~nm}$ on a Jasco FP-8200 spectrofluorometer (Jasco Corporation, 169 Japan). For the CR binding assay, incubated proteins were prepared at 170 a final concentration of $20 \mu \mathrm{M}$ and $\mathrm{CR}$ was mixed to a final 171 concentration of $20 \mu \mathrm{M}$. Absorption spectra were recorded in the 172 range from 375 to $700 \mathrm{~nm}$ in a Specord 200 Plus spectrophotometer 173 (Analytik Jena, Germany). Spectra of protein alone and buffer were 174 acquired to subtract their contribution to the signal.

Transmission Electron Microscopy (TEM). For TEM sample 176 preparation, $10 \mu \mathrm{L}$ of the incubated proteins was deposited on a 177 carbon-coated copper grid for $10 \mathrm{~min}$, and the excess liquid was 178 removed with filter paper, followed by negative staining with $10 \mu \mathrm{L}$ of 179 $2 \%(\mathrm{w} / \mathrm{v})$ uranyl acetate for $1 \mathrm{~min}$. Grids were scanned using a JEM 180 1400 transmission electron microscope (JEOL Ltd., Japan) operating 181 at $80 \mathrm{kV}$, and images were acquired with a CCD GATAN ES1000W 182 Erlangshen camera (Gatan Inc.). The particles' diameter was analyzed 183 with Image J software (National Health Institute), averaging the 184 measures of 100 individual particles.

Scanning Electron Microscopy (SEM). A sample of $50 \mu \mathrm{L}$ of 186 incubated protein was centrifuged, and the resulting supernatant was 187 removed. The precipitate was resuspended in $50 \mu \mathrm{L}$ of water and 188 washed twice to remove salt traces. Then, $5 \mu \mathrm{L}$ of the resuspension 189 was deposited on a silicon slice $(0.5 \mathrm{~cm} \times 0.5 \mathrm{~cm})$ and dried with 190 nitrogen flow. Silicon slices were scanned using a Merlin field- 191 emission scanning electron microscope (Zeiss Ltd., Germany) at 2192 $\mathrm{kV}$, and images were acquired with an in-lens SE detector. The 193 diameter of the particles was calculated with Image J (National Health 194 Institute), averaging the measures of 100 individual particles. 195

Fourier Transform Infrared (FTIR) Spectroscopy. DHFR-wt 196 was dissolved at $100 \mu \mathrm{M}$ in PBS, $\mathrm{pH}$ 7.4, and filtered through a 0.22197 $\mu \mathrm{m}$ filter. Then, $30 \mu \mathrm{L}$ of the incubated proteins were centrifuged at 198 $12000 \mathrm{~g}$ for $30 \mathrm{~min}$ and resuspended in $10 \mu \mathrm{L}$ of water. Samples were 199 placed on the attenuated total reflectance (ATR) crystal and dried out 200 
201 under a $\mathrm{N}_{2}$ flow. The experiments were carried out in a Bruker Tensor 20227 FTIR (Bruker Optics) supplied with a Specac Golden Gate MKII 203 ATR accessory. Each spectrum consists of 32 acquisitions measured at 204 a resolution of $2 \mathrm{~cm}^{-1}$ using the three-term Blackman-Harris 205 Window apodization function. Data was acquired and normalized 206 using OPUS MIR Tensor 27 software (Bruker Optics), with the Min/ 207 Max normalization method, which scales spectrum intensities to the 208 effect that the minimum absorbance unit will be 0 and the maximum 209 will be 2 . Infrared (IR) spectra were fitted employing a nonlinear 210 peak-fitting equation using Origin 8.5 (OriginLab Corporation).

211 Fluorescence Microscopy. First, $20 \mu \mathrm{L}$ of incubated fusion 212 proteins was centrifuged at $12000 \mathrm{~g}$ for $30 \mathrm{~min}$, and the supernatant 213 was removed. The precipitate was resuspended in PBS $\mathrm{pH} 7.4$ 214 containing $100 \mu \mathrm{M}$ fMTX (methotrexate labeled with fluorescein, 215 ThermoFisher Scientific) and incubated for $30 \mathrm{~min}$. For the 216 bifunctional fusion protein Sup35-5aa-DHFR-Z, the precipitate was 217 resuspended in PBS pH 7.4 containing $100 \mu \mathrm{M}$ fMTX and $1 \mu \mathrm{g}$ of 218 anti-EGFR antibody labeled with Alexa 555 (ThermoFisher 219 Scientific) and incubated for $30 \mathrm{~min}$. Samples were washed three 220 times to remove any unbound fMTX and antibody and then 221 resuspended in PBS to a final volume of $50 \mu \mathrm{L}$. Then, $5 \mu \mathrm{L}$ of the 222 resuspension was dropped onto a clean glass slide (Deltalab, $26 \mathrm{~mm} \times$ $22376 \mathrm{~mm}$ ) and covered by a cover slide (Deltalab, $22 \mathrm{~mm} \times 22 \mathrm{~mm}$ ). 224 Fluorescence imaging of particles and fibrils was carried out on an 225 Eclipse 90i epifluorescence optical microscope equipped with a Nikon 226 DXM1200F (Nikon, Japan) camera and ACT-1 software. Images 227 were acquired with an excitation filter of $465-495 \mathrm{~nm}$ or $540-580$ $228 \mathrm{~nm}$ and detecting fluorescence emission in a range of $515-555 \mathrm{~nm}$ or $229590-630 \mathrm{~nm}$.

230 Dynamic Light Scattering (DLS). The size of the incubated 231 proteins was determined using a Malvern Zetasizer Nano ZS90 (ATA 232 Scientific, Australia) in PBS buffer, $\mathrm{pH} 7.4$, at $25^{\circ} \mathrm{C}$.

233 Catalytic Activity of Dihydrofolate Reductase Embedded in 234 Amyloid Assemblies. The Sup35-5aa-DHFR particles and Sup352358 aa-DHFR fibrils were prepared at $2 \mu \mathrm{M}$ in an activity buffer $(0.1 \mathrm{M}$ 236 potassium phosphate, $\mathrm{pH} 7.4,1 \mathrm{mM}$ DTT, $0.5 \mathrm{M} \mathrm{KCl}, 1 \mathrm{mM}$ 237 ethylenediaminetetraacetic acid (EDTA), $20 \mathrm{mM}$ sodium ascorbate). 238 Then, $50 \mu \mathrm{L}$ of the sample was mixed with $850 \mu \mathrm{L}$ of activity buffer in 239 a cuvette and preincubated at room temperature for $10 \mathrm{~min}$. The 240 reaction was then initiated by adding $50 \mu \mathrm{L}$ of $2 \mathrm{mM} \mathrm{7,8-}$ 241 dihydrofolate (DHF) solution and $50 \mu \mathrm{L}$ of a $2 \mathrm{mM}$ nicotinamide 242 adenine dinucleotide phosphate (NADPH) solution. Sup35-SAC 243 fibrils and lysozyme were used as negative controls. For the 244 determination of the kinetic parameters, a final concentration of 20 $245 \mathrm{nM}$ assemblies and $20 \mu \mathrm{M}$ of DHF was preincubated in an activity 246 buffer at room temperature. The reaction was initiated by adding $247 \mathrm{NADPH}$ to a final concentration in the range from 10 to $200 \mu \mathrm{M}$. The 248 absorbance change at $340 \mathrm{~nm}$ was monitored on a Specord 200 Plus 249 spectrophotometer (Analytik Jena, Germany). The initial velocity at 250 each concentration was determined upon fitting the reaction with 251 Origin 8.5 (OriginLab Corporation). The reciprocal of the velocity of 252 the reaction was plotted against the reciprocal of the concentration of $253 \mathrm{NADPH}$. The catalytic constants $V_{\max }, K_{\mathrm{m}}, K_{\text {cat }}$ and $K_{\text {cat }} / K_{\mathrm{m}}$ were 254 calculated.

255 Thermodynamic Dissociation Constants. The equilibrium 256 dissociation constants $\left(K_{\mathrm{d}}\right)$ for enzyme-NADPH complexes were 257 determined by fluorescence titration at $25{ }^{\circ} \mathrm{C}$. In particular, the 258 Sup35-5aa-DHFR and Sup35-8aa-DHFR assemblies were prepared at $259100 \mathrm{nM}$ in an activity buffer in a quartz cuvette. The titrations were 260 carried out by serial additions of aliquots $(1-4 \mu \mathrm{L})$ of NADPH into 261 the cuvette. The intrinsic fluorescence spectra in the range from 300 262 to $400 \mathrm{~nm}$ were recorded $2 \mathrm{~min}$ after the addition of NADPH, using 263 an excitation wavelength of $290 \mathrm{~nm}$ and an emission bandwidth of 5 $264 \mathrm{~nm}$ in a Jasco FP-8200 spectrofluorometer (Jasco Corporation, 265 Japan). A standard tryptophan solution was titrated, and the emission 266 was used to correct for the inner filter effect caused by NADPH. The 267 change in intrinsic fluorescence emission at $340 \mathrm{~nm}$ was plotted 268 against the NADPH concentration using Origin 8.5 (OriginLab 269 Corporation), and the $K_{\mathrm{d}}$ was calculated from the binding equation.
Preparation of fMTX- or/and Antibody-Loaded Nano- 270 particles. The incubated protein Sup35-DHFR-Z was precipitated 271 by centrifugation at $13000 \mathrm{rpm}$ for $30 \mathrm{~min}$. The precipitate was 272 resuspended in PBS buffer $\mathrm{pH} 7.4$ containing $1 \mu \mathrm{g}$ of antibody or/and 273 $100 \mu \mathrm{M}$ fMTX (fluorescein-labeled MTX) and then incubated for 30274 min. The concentration of nanoparticles was determined by the 275 reduction of absorbance at $280 \mathrm{~nm}$ in the supernatant fraction. Three 276 labeling antibodies (ThermoFisher Scientific) - anti-EGFR antibody 277 labeled with Alexa fluor 555, anti-CD3 antibody labeled with Alexa 278 fluor 488, and goat antirabbit antibody labeled with Alexa fluor 555- 279 were used in this study. Then, the antibody or/and fMTX conjugated 280 particles were washed three times with PBS buffer to remove any 281 unbound antibody or/and fMTX and resuspended in PBS buffer. The 282 antibody-loaded nanoparticles (NPs-anti-EGFR and NPs-anti-CD3), 283 fMTX-loaded nanoparticles (NPs-fMTX), and fMTX- and antibody- 284 loaded nanoparticles (NPs-fMTX-anti-EGFR and NPs-fMTX-anti- 285 rabbit) were used for experiments immediately.

Cell Culture. The human HeLa and nontumoral lung MRC-5 cell 287 lines were obtained from the American Type Culture Collection 288 (ATCC). Both cells were maintained in minimum essential medium $\alpha 289$ media (MEM- $\alpha$ ), supplemented with $10 \%$ fetal bovine serum (FBS), 290 and incubated at $37^{\circ} \mathrm{C}$ with $5 \% \mathrm{CO}_{2}$.

291

Cytotoxicity Assay. HeLa and MRC-5 cells were seeded on a 96- 292 well plate at a concentration of $3 \times 10^{3} /$ well and incubated at $37{ }^{\circ} \mathrm{C} 293$ for $24 \mathrm{~h}$. For HeLa cells, the medium was replaced with $100 \mu \mathrm{L}$ of 294 fresh medium containing the treatments. Unloaded Sup35-5aa- 295 DHFR-Z particles were assayed in a range of concentrations $0.5-296$ $60 \mu \mathrm{M}$. MTX-loaded nanoparticles (NPs-MTX), MTX- and anti- 297 EGFR antibody-loaded nanoparticles (NPs-MTX-anti-EGFR), and 298 free MTX were assayed in a concentration range of $2-20 \mu \mathrm{M}$. For 299 NPs-MTX-anti-EGFR samples, the medium was removed, and cells 300 were rinsed three times with DPBS buffer after incubation of $20 \mathrm{~min} .301$ Then, a fresh medium was added. PBS alone was used as the vehicle 302 control and the medium without cells as a blank. A final concentration 303 of $5 \mathrm{ng} \cdot \mathrm{mL}^{-1}$ proteinase $\mathrm{K}$ was added to the medium to mimic the 304 proteinase-enriched microenvironment of tumor tissues. For MRC-5 305 cells, the medium was replaced with a fresh medium containing 2-20 306 $\mu \mathrm{M}$ Sup35-5aa-DHFR-Z nanoparticles, followed by addition of 307 proteinase $\mathrm{K}$ to a final concentration of $5 \mathrm{ng} \cdot \mathrm{mL}^{-1}$. Each sample 308 was measured in triplicate, and the plate was incubated at $37^{\circ} \mathrm{C}$ with 309 $5 \% \mathrm{CO}_{2}$ for $48 \mathrm{~h}$. Then, $10 \mu \mathrm{L}$ of the PrestoBlue cell viability reagent 310 (ThermoFisher Scientific) was added to each well and incubated for 311 another $1 \mathrm{~h}$. The fluorescence emission was analyzed on a Victor III 312 Multilabel Plate Reader (Perkin Elmer), equipped with a 530/10 nm 313 CW-lamp filter and a 590/20 nm emission filter. The viability of cells 314 was calculated as follows:

$$
\text { viability }(\%)=\left(I_{\text {test }}-I_{\text {blank }}\right) /\left(I_{\text {control }}-I_{\text {blank }}\right) \times 100 \%
$$

where $I_{\text {test }} I_{\text {blank }}$ and $I_{\text {control }}$ are the fluorescence intensities of the test, 316 blank, and control groups, respectively. The significance of the 317 differences between the test groups and the control was analyzed by 318 one-way analysis of variance (ANOVA) using the Origin 8.5 program 319 (OriginLab Corporation).

Confocal Microscopy. HeLa cells were cultured on an 8-well 321 Millicell EZ slide (Millipore, Germany) to a final confluence of 70- 322 $80 \%$. Then, the medium was replaced with a fresh medium containing 323 $20 \mu \mathrm{M}$ NPs-anti-EGFR and NPs-fMTX-anti-EGFR. The slide was 324 incubated at $37{ }^{\circ} \mathrm{C}, 5 \% \mathrm{CO}_{2}$ for $20 \mathrm{~min}$. The anti-CD3 IgG-loaded 325 nanoparticles (NPs-anti-CD3) were used as a control. For the 326 internalization assay, the medium was replaced with a fresh medium 327 containing $10 \mu \mathrm{M}$ Free fMTX and fMTX-loaded nanoparticles (NPs- 328 fMTX) preincubated with $50 \mathrm{ng} \cdot \mathrm{mL}^{-1}$ proteinase for $5 \mathrm{~min}$ followed 329 by addition of EDTA. The slide was incubated at $37^{\circ} \mathrm{C}, 5 \% \mathrm{CO}_{2}$ for 4330 h. Then, the medium was removed. The adherent cells on the slide 331 were rinsed three times with a fresh medium. Cells were stained with 332 CellMask Deep Red (Invitrogen, ThermoFisher Scientific) at a final 333 dilution of 1:1000 for $10 \mathrm{~min}$. The medium was removed and washed 334 three times with PBS buffer. Cells were fixed with $4 \% 335$ paraformaldehyde (PFA) at room temperature for $20 \mathrm{~min}$, followed 336 by a washing step with PBS buffer. Then, $10 \mu \mathrm{L}$ of the mounting 337 
338 medium containing 4',6-diamidino-2-phenylindole (DAPI) was 339 dropped onto each well of the slide and covered with a coverslip. 340 The slide was imaged on a Leica TCS SP5 confocal microscope 341 (Leica Biosystems, Germany). Images were acquired using 405, 488 $342 \mathrm{~nm}$, or 561 and $633 \mathrm{~nm}$ excitation laser for DAPI, Alexa fluor 488, or $343555 \mathrm{~nm}$ and CellMask Deep Red, respectively.

344 Flow Cytometry Assay. The NPs-fMTX-anti-EGFR, NPs-fMTX, 345 and NPs-fMTX-antirabbit were prepared as described above. HeLa 346 cells were prepared in PBS buffer $\mathrm{pH} 7.4$ at a final concentration of 1 $347 \times 10^{6} \mathrm{~mL}^{-1}$. Then, $200 \mu \mathrm{L}$ of HeLa cells was precipitated and 348 resuspended in $200 \mu \mathrm{L}$ of PBS buffer containing $20 \mu \mathrm{M}$ NPs-fMTX349 anti-EGFR, NPs-fMTX, and NPs-fMTX-antirabbit, respectively. After $35030 \mathrm{~min}$ of incubation, the cells were pelleted and washed three times. 351 Then, $200 \mu \mathrm{L}$ of the cell suspension was analyzed using a 352 FACSCalibur cytometer (BD Biosciences, Becton Dickinson), 353 equipped with a FITC laser. Fluorescence intensities of cell-bound 354 nanoparticles were analyzed and quantitated using FlowJo (BD 355 Biosciences). Cells treated with PBS were used as the control.

Proteinase Digestion of fMTX-Loaded Sup35-DHFR-Z 357 Particles. The fMTX-loaded particles (NPs-fMTX) were resus358 pended in $\mathrm{PBS}$ buffer containing proteinase $\mathrm{K}$ at a final concentration 359 of $1 \mu \mathrm{g} \cdot \mathrm{mL}^{-1}$. SDS-PAGE was used to analyze the proteolytic progress 360 at 15,30 , and $45 \mathrm{~min}$. The supernatant's fluorescence spectrum was 361 recorded in the $500-600 \mathrm{~nm}$ range at different time points, using an 362 excitation wavelength of $488 \mathrm{~nm}$ and an emission bandwidth of $5 \mathrm{~nm}$ 363 on a Jasco FP-8200 spectrofluorometer (Jasco Corporation, Japan). 364 The emission maximum was plotted as a function of time. The 365 experiment was performed in duplicate. Lysozyme and PBS, instead of 366 proteinase $\mathrm{K}$, were used as negative controls.

\section{RESULTS AND DISCUSSION}

368 Self-Assembly of Sup35-8aa-DHFR into Active Amy369 loid Fibrils. To generate functional amyloid fibrils with a 370 previously unexplored catalytic activity, we fused Sup35-SAC 371 to the E. coli DHFR through an 8-residue-long flexible linker 372 consisting of $\mathrm{SG}_{3} \mathrm{SG}_{2} \mathrm{~S}$ (Sup35-8aa-DHFR) (Figure S1A,B). 373 The size of DHFR $(21.5 \mathrm{kDa})$ is in the range of GFP $(27 \mathrm{kDa})$ 374 and carbonic anhydrase $(30 \mathrm{kDa})$, two proteins that assemble 375 into active amyloid fibrils when fused to Sup35-SAC.

376 The fusion protein, Sup35-8aa-DHFR, was expressed 377 recombinantly at $64 \mathrm{mg} \cdot \mathrm{L}^{-1}$ and located in the soluble cellular 378 fraction, from which we purified it (Figure S1C). We 379 compared the conformational properties of soluble wild-type 380 DHFR (DHFR-wt) and Sup35-8aa-DHFR proteins by far-UV 381 circular dichroism (CD) (Figure 1A). Their spectra were 382 virtually identical, indicating that Sup35-SAC does not induce 383 significant changes in the DHFR structure. Then, we used the 384 amyloid-specific dyes Thioflavin- $\mathrm{T}$ (Th-T) and Congo Red 385 (CR) to assess if, despite its solubility, Sup35-8aa-DHFR self386 assembles with time into amyloid-like structures. To this aim, 387 we incubated the fusion protein in PBS buffer $\mathrm{pH} 7.4$ at $37{ }^{\circ} \mathrm{C}$ 388 for four days. The incubated Sup35-8aa-DHFR protein 389 promoted a substantial increase in the $\mathrm{Th}-\mathrm{T}$ fluorescence 390 emission signal, whereas DHFR-wt incubated in the same 391 conditions had a negligible effect (Figure 1B). In agreement 392 with these results, CR binding was observed for incubated 393 Sup35-8aa-DHFR, resulting in a red shift of the CR absorption 394 spectrum, while DHFR-wt did not promote any CR spectral 395 change (Figure 1C). Moreover, the Fourier transform infrared 396 (FTIR) absorbance spectrum of incubated Sup35-8aa-DHFR 397 in the amide I region evidenced the existence of a band at 1621 $398 \mathrm{~cm}^{-1}$, which can be assigned to the intermolecular $\beta$-sheet 399 structure characteristic of amyloids (Figure 1D and Table S1). 400 This band was absent in the FTIR spectrum of incubated 401 DHFR-wt (Figure S3 and Table S1). The morphological 402 analysis of the two protein solutions by negative staining and
A
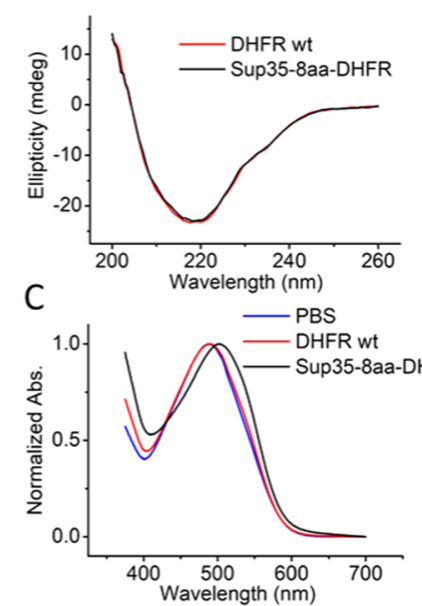

E

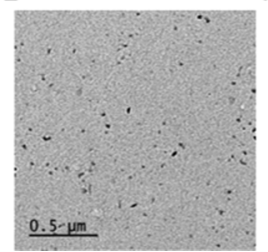

F

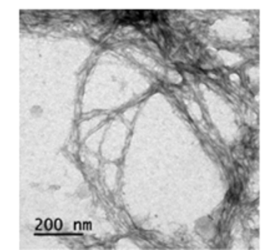

G

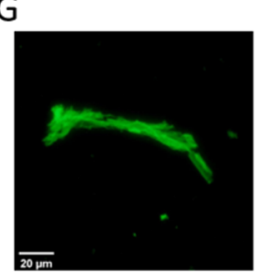

Figure 1. Characterization of soluble and aggregated Sup35-8aaDHFR. (A) Far-UV CD spectra of soluble Sup35-8aa-DHFR and DHFR-wt, shown in black and red, respectively. Incubated Sup35-8aaDHFR was analyzed by measuring the Th-T fluorescence emission (B) and CR absorbance (C). DHFR-wt and Sup35-8aa-DHFR are shown in red and black, respectively. PBS without protein was included as a control (blue line). (D) FTIR absorbance spectra of incubated Sup35-8aa-DHFR in the amide I region of the spectrum (solid black line) and the component bands (dashed lines); the intermolecular $\beta$-sheet component is shown in blue. (E, F) Representative TEM micrographs of incubated proteins upon negative staining: (E) DHFR-wt and (F) Sup35-8aa-DHFR. Scale bar represents 500 and $200 \mathrm{~nm}$, respectively. (G) Fluorescence microscopy image of Sup35-8aa-DHFR fibrils incubated with fMTX. Scale bar represents $20 \mu \mathrm{m}$.
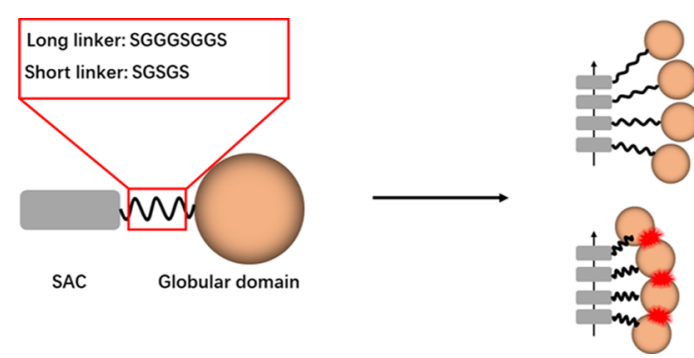

Fibrils

Figure 2. Schematic illustration of the rationale for the formation of stable amyloid oligomers. The scheme of SAC (gray box) is connected with a globular domain (orange ball) by linkers of different lengths. Molecules with long linkers can form infinite amyloid fibrils, whereas those with short linkers would form preferably oligomers, owing to the steric restriction imposed by adjacent globular domains.

transmission electron microscopy (TEM) indicated that the 403 incubated fusion protein assembled into a typical fibrillar 404 amyloid-like structure, whereas DHFR-wt did not form any 405 kind of assembly (Figure 1E,F). About $36.1 \pm 4.2 \%$ of the 406 original monomer in the solution was incorporated into fibrils 407 at the end of the incubation reaction. The average size of the 408 fibrils is $955 \pm 75 \mathrm{~nm}$, as measured by DLS. This is just a 409 
A

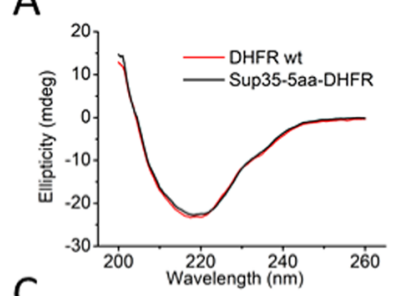

C

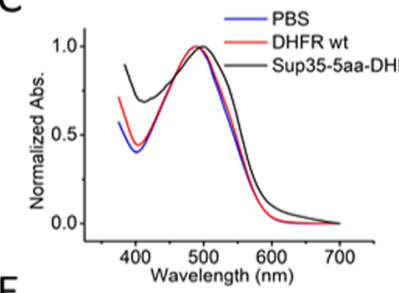

$\mathrm{E}$

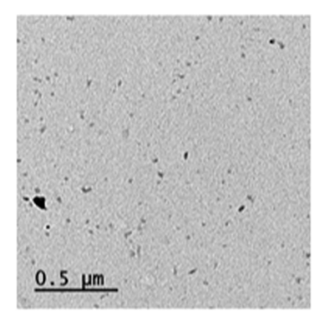

G

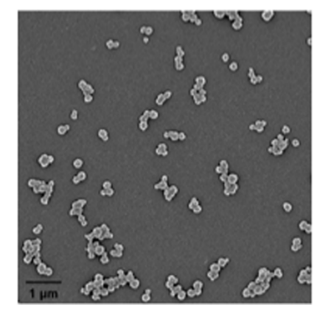

B
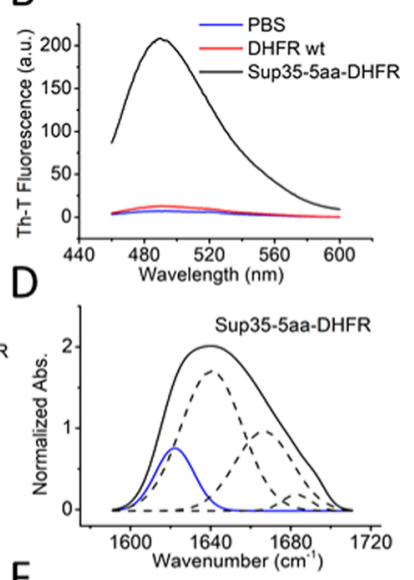

F

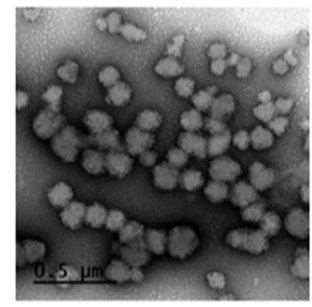

$\mathrm{H}$

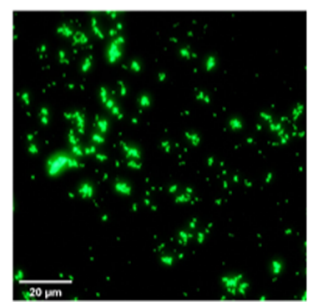

Figure 3. Characterization of soluble and aggregated Sup35-5aaDHFR. (A) Far-UV CD spectra of soluble Sup35-5aa-DHFR and DHFR-wt, shown in black and red, respectively. Incubated Sup35-5aaDHFR was analyzed by measuring Th-T fluorescence emission (B) and CR absorbance (C). DHFR-wt and Sup35-5aa-DHFR are shown in red and black, respectively. PBS without protein was included as a control (blue line). (D) FTIR absorbance spectra of incubated Sup358 aa-DHFR in the amide I region of the spectrum (solid black line) and the component bands (dashed lines); the intermolecular $\beta$-sheet component is shown in blue. (E, F) Representative TEM micrographs of incubated proteins upon negative staining: (E) DHFR-wt and (F) Sup35-5aa-DHFR. Scale bar represents $500 \mathrm{~nm}$. (G) Representative SEM micrograph of Sup35-5aa-DHFR particles. Scale bar represents 1 $\mu \mathrm{m}$. (H) Fluorescence microscopy image of Sup35-5aa-DHFR particles incubated with fMTX. Scale bar represents $20 \mu \mathrm{m}$.

410 rough estimation of the fibrils' dimensions, since they are 411 nonspherical particles.

412 To test if the DHFR enzyme keeps the native structure 413 within the observed amyloid fibrils, we used a fluorescein414 labeled version of MTX (fMTX), a competitive inhibitor that 415 binds to the active site of DHFR. In the presence of the 416 inhibitor, the amyloid fibrils appeared green, as visualized by 417 fluorescence microscopy (Figure 1G), indicating that fMTX 418 can bind to the catalytic site of the DHFR moieties embedded 419 in these nanostructures.

420 Self-Assembly of Sup35-5aa-DHFR into Amyloid 421 Oligomeric Nanoparticles. As shown above, a linker of 422 eight residues suffices to allow the formation of infinite fibrils 423 in which DHFR remains in its native conformation (Figure 2). 424 Our recently derived model predicts that introducing a shorter

A

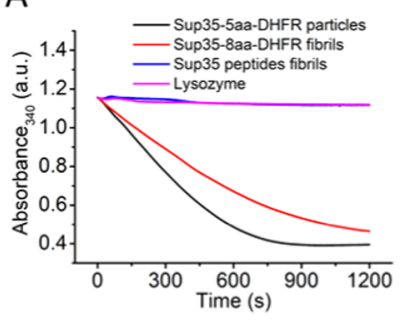

B

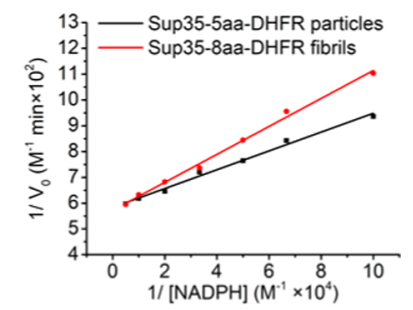

C

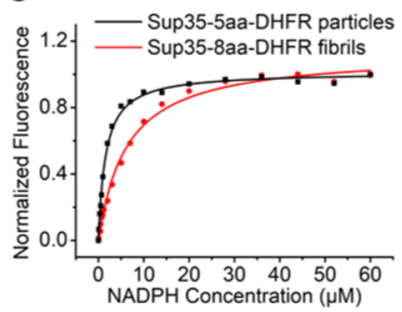

Figure 4. Catalytic activity and NADPH binding affinity of Sup355aa-DHFR particles and Sup35-8aa-DHFR fibrils. (A) The change in absorbance at $340 \mathrm{~nm}$ was monitored for each sample for $20 \mathrm{~min}$ in the presence of DHF and NADPH. Both assemblies were prepared at $100 \mathrm{nM}$. Sup35-SAC peptide fibrils and lysozyme are negative controls. (B) Lineweaver-Burk plot obtained by plotting the reciprocal of initial velocities of reactions against the reciprocal of the concentration of NADPH. (C) Measurement of $K_{\mathrm{d}}$ for NADPH binding to both assemblies by monitoring the quenching of DHFR intrinsic fluorescence. Fluorescence titrations were performed using $100 \mathrm{nM}$ assemblies and the indicated concentrations of NADPH.

linker to connect the amyloidogenic and globular moieties 425 would generate steric hindrance and might result in the 426 formation of oligomeric structures, instead of fibrils (Figure 427 2). ${ }^{16}$ To experimentally demonstrate this hypothesis, we 428 constructed a Sup35-5aa-DHFR fusion, in which we connected 429 Sup35-SAC and DHFR with a 5-residue linker consisting of 430 SGSGS (Figure S2A,B).

431

Again, the protein was well-expressed $\left(71 \mathrm{mg} \cdot \mathrm{L}^{-1}\right)$ and 432 soluble (Figure S2C). Purified Sup35-5aa-DHFR displayed a 433 conformation very similar to that of DHFR-wt in solution, as 434 assessed by far-UV CD (Figure 3A). The protein was $435 \mathrm{f3}$ incubated at $37{ }^{\circ} \mathrm{C}$ for four days, rendering $\mathrm{Th}-\mathrm{T}$ (Figure 436 $3 \mathrm{~B}$ ) and CR (Figure 3C) positive assemblies, with detectable 437 intermolecular $\beta$-sheet contents (Figure $3 \mathrm{D}$ and Table S1). 438 TEM imaging evidenced that, despite these amyloid-like 439 properties, and in contrast to Sup35-8aa-DHFR, Sup35-5aa- 440 DHFR assembles into spherical nanoparticles, which is 441 consistent with an amyloid oligomeric nature (Figure 3E,F). 442 The presence of spherical structures in the incubated Sup35- 443 5aa-DHFR solution was further confirmed by scanning 444 electron microscopy (SEM) (Figure 3G). About $40.2 \pm 445$ $3.3 \%$ of the original monomer in the solution was incorporated 446 into nanoparticles at the end of the incubation reaction. 447

The particles were very homogenous in size, with diameters 448 of $147 \pm 20$ and $159 \pm 24 \mathrm{~nm}$ as measured by TEM and SEM, 449 respectively. These sizes are in good agreement with the $150 \pm 450$ $50 \mathrm{~nm}$ measured for the particles in solution by dynamic light 451 scattering (DLS) (Figure S4). Incubation of this sample with 452 fMTX rendered green fluorescent aggregates (Figure $3 \mathrm{H}$ ), 453 indicating that, as in the amyloid fibrils, the inhibitor can bind 454 to folded DHFR domains in the structure of the oligomeric 455 protein nanoparticles. Therefore, as predicted, playing with the 456 linker length allowed us to tune the assemblies' mesoscopic 457 

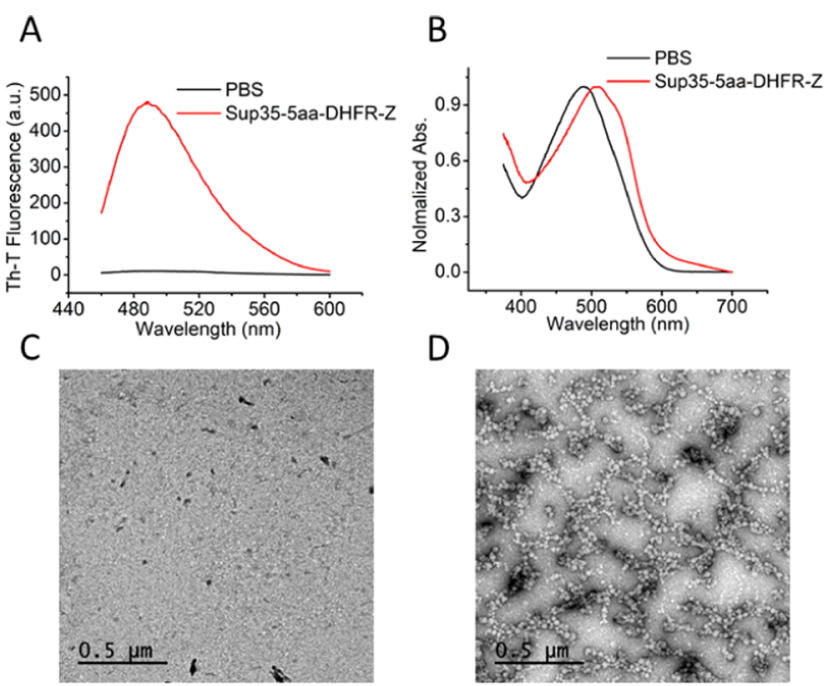

\section{D}

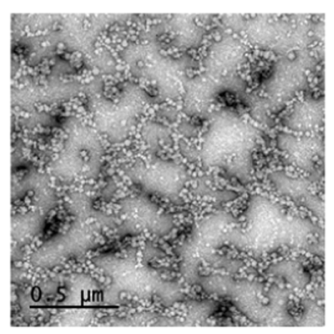

$\mathrm{E}$

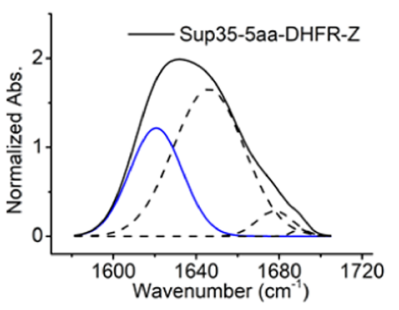

$\mathrm{F}$

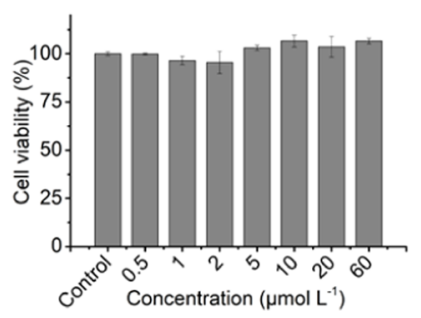

Figure 5. Characterization of aggregated Sup35-5aa-DHFR-Z. Incubated Sup35-5aa-DHFR-Z was analyzed by measuring the Th$T$ fluorescence emission (A) and CR absorbance (B). Sup35-5aaDHFR-Z is shown in red; PBS without protein was included as a control (black line). Representative TEM micrographs of soluble Sup35-5aa-DHFR-Z (C) and incubated Sup35-5aa-DHFR-Z. (D) Scale bar represents $500 \mathrm{~nm}$. (E) FTIR absorbance spectra of incubated Sup35-5aa-DHFR-Z in the amide I region of the spectrum (solid black line) and the component bands (dashed lines); the intermolecular $\beta$-sheet component is shown in blue. (F) Cytotoxicity of incubated Sup35-5aa-DHFR-Z. Results are expressed as means \pm standard deviation (SD), $n=3$, and analyzed using a one-way ANOVA test. The statistical differences between the control group and the test group were established at $P<0.05$.

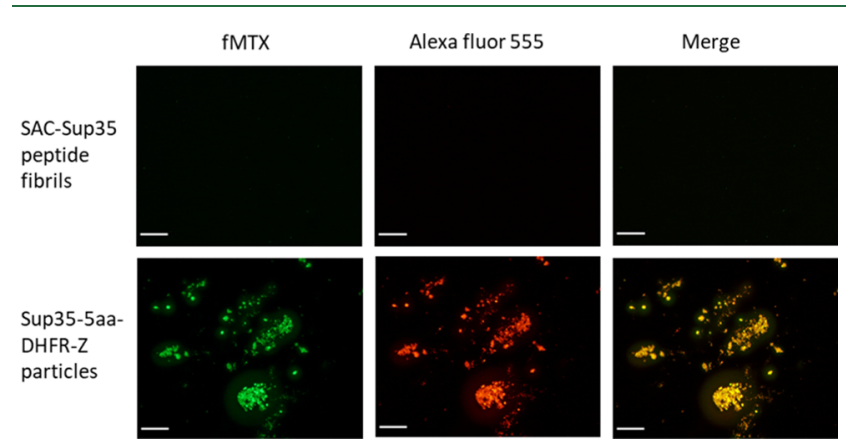

Figure 6. Dual-binding activity of Sup35-5aa-DHFR-Z nanoparticles. Representative fluorescence microscopy images of SAC-Sup35 peptide fibrils (upper panel) and Sup35-5aa-DHFR-Z nanoparticles (lower panel) incubated with fMTX (fluorescein, green channel) and an anti-EGFR antibody (Alexa fluor 555, red channel). Scale bar represents $50 \mu \mathrm{m}$.

458 properties and produce, for the first time, amyloid-like active 459 spherical nanoparticles.
Catalytic Activity of DHFR in Fibrils and Nano- 460 particles. DHFR catalyzes the reduction of dihydrofolate 461 (DHF) to tetrahydrofolate (THF) in the presence of reduced 462 nicotinamide adenine dinucleotide phosphate (NADPH). ${ }^{27} 463$ The binding of fMTX to fibrils and nanoparticles suggested 464 that DHFR might keep its enzymatic activity in both kinds of 465 assemblies. To further confirm this extent and evaluate the 466 impact of each superstructure on the activity of the enzyme, we 467 measured the catalytic activity of fibrils and nanoparticles at 468 $100 \mathrm{nM}$ in the presence of DHF and NADPH. We recorded 469 the absorbance change at $340 \mathrm{~nm}$ for $20 \mathrm{~min}$ and plotted the 470 resulting traces (Figure 4A). For both fibrils and nanoparticles, $471 \mathrm{f} 4$ the decrease of NADPH absorbance with time indicates that 472 they are catalytically active, whereas the fibrils formed by the 473 Sup35-SAC peptide alone or lysozyme, used as negative 474 controls, do not affect the NADPH absorbance at $340 \mathrm{~nm} .475$ Importantly, the signal decreased faster for the nanoparticles 476 than for the fibrils, which suggested that the smaller assemblies 477 were more active, assuming that the proportion of properly 478 folded DHFR moieties is similar in both assemblies.

479

Next, we sought to determine the apparent kinetic constants 480 for both amyloid-like assemblies. We measured the initial 481 velocities of the reactions in the presence of $\mathrm{NADPH}$ in a 482 range of concentrations from 10 to $200 \mu \mathrm{M}$, while the 483 concentration of DHF was fixed at $20 \mu \mathrm{M}$. Then, we calculated 484 the kinetic constants from the resulting Lineweaver-Burk plots 485 (Figure 4B and Table S2). The nanoparticles' $V_{\max }$ was $1712 \pm 486$ $25 \mathrm{nM} \cdot \mathrm{min}^{-1}$, which does not differ significantly from that of 487 the fibrils $\left(V_{\max }=1745 \pm 21 \mathrm{nM} \cdot \mathrm{min}^{-1}\right)$. This was expected 488 since the NADPH binding site and the catalytic center of 489 DHFR should keep the same conformation in both assemblies. 490 However, the nanoparticles exhibited a lower $K_{\mathrm{m}}(6.23 \pm 0.32491$ $\mu \mathrm{M})$ than that of the fibrils $(9.44 \pm 0.41 \mu \mathrm{M})$. This translates 492 into a higher specificity constant for the nanoparticles $\left(K_{\text {cat }} / K_{\mathrm{m}} 493\right.$ $\left.=0.23 \pm 0.01 \mu \mathrm{M}^{-1} \cdot \mathrm{s}^{-1}\right)($ Table S2).

The thermodynamic dissociation constants $\left(K_{\mathrm{d}}\right)$ for binding 495 of NADPH to both assemblies were measured by monitoring 496 the quenching of the DHFR intrinsic fluorescence (Figure 4C). 497 The obtained values indicated that the binding affinity of 498 nanoparticles for NADPH $\left(K_{d}=1.58 \pm 0.07 \mu \mathrm{M}\right)$ was 4 -fold 499 higher than that of the fibrils, with a $K_{d}$ value of $6.26 \pm 0.44500$ $\mu \mathrm{M}$ (Table S2). Thus, both the $K_{\mathrm{m}}$ and $K_{\mathrm{d}}$ constants indicate 501 that the higher catalytic activity of the nanoparticles results 502 from a higher NADPH binding capability of DHFR when 503 embedded in the spherical amyloid-like structures, likely 504 because the cofactor binding site is more accessible in these 505 assemblies or, alternatively, because the proportion of 506 conformationally active DHFR molecules is higher in the 507 particles than in the fibrils.

Overall, the activity, homogeneity, stability, spherical 509 structure, and moderate size of the novel oligomeric 510 amyloid-like nanoparticles we describe here seem optimal for 511 their exploitation as functionalized nanomaterials. 512

Building Up Sup35-5aa-DHFR-Z Bifunctional Nano- 513 particles. We sought to exploit the ability of Sup35-5aa- 514 DHFR to form spherical structures of defined size to build up 515 bifunctional nanoparticles with potential applications in 516 targeted delivery. To this aim, we incorporated the Z-domain 517 of $S$. aureus protein A ( $\mathrm{SpA})$ at the C-terminus of Sup35-5aa- 518 DHFR through a flexible $S_{2} S G$ linker, to obtain the Sup35- 519 5aa-DHFR-Z tripartite fusion protein (Figure S5A,B). The Z- 520 domain is an engineered analog of the B-domain, one of the 521 five homologous IgG-binding domains of SpA. ${ }^{28}$ It consists of 522 
A
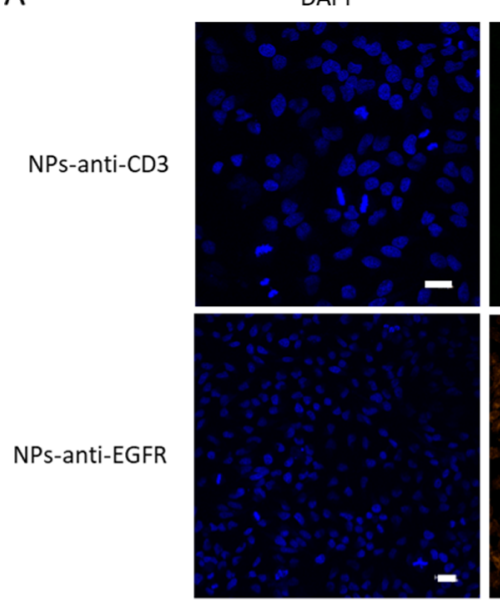

B
Alexa 488 or 555
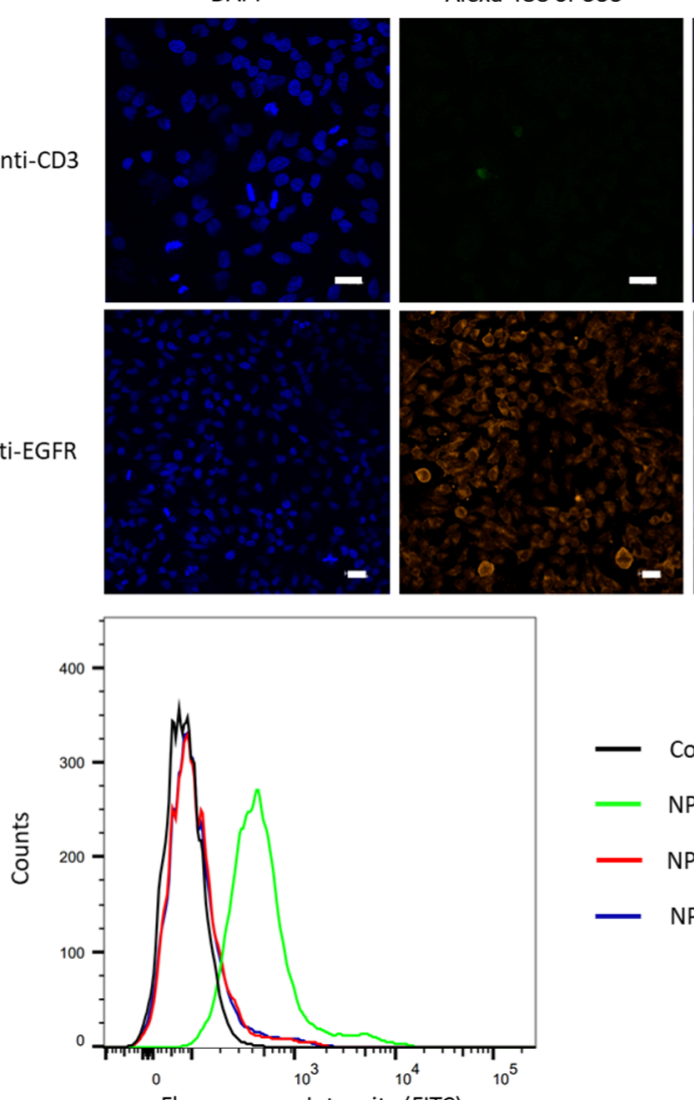

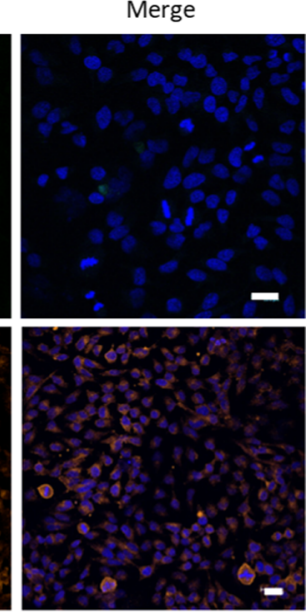

Merge

- Control

NPs-fMTX-anti-EGFR

- NPs-fMTX-anti-Rabbit

- NPs-fMTX

Figure 7. Binding specificity of functionalized Sup35-5aa-DHFR-Z nanoparticles to HeLa cells. (A) Representative confocal microscopy images of HeLa cells incubated with nanoparticles conjugated with an anti-CD3 antibody (NPs-anti-CD3, Alexa 488) (upper panel) or an anti-EGFR antibody (NPs-anti-EGFR, Alexa 555) (lower panel). Scale bar represents $20 \mu \mathrm{m}$. (B) Quantitative analysis of fluorescein fluorescence on HeLa cells by flow cytometry. HeLa cells were incubated with fMTX, anti-EGFR-loaded nanoparticles (NPs-fMTX-anti-EGFR, green line), fMTX and goat antirabbit antibody-loaded nanoparticles (NPs-fMTX-antirabbit, red line), and fMTX-loaded nanoparticles (NPs-fMTX, blue line). HeLa cells treated with PBS were used as a control. Fluorescein-labeled MTX (fMTX) was used as a fluorescence probe to calculate the proportion of cancer cells bound to nanoparticles using an FITC detector.

52358 residues $(6.5 \mathrm{kDa})$ and folds into a bundle-like shape 524 composed of three $\alpha$-helices. The Z-domain binds with high 525 affinity to the Fc region of antibodies from different species 526 and subclasses. The idea was that if Sup35-5aa-DHFR-Z 527 assembles into amyloid nanoparticles, we might decorate them 528 with any antibody of interest.

529 Once more, the protein was well-expressed $\left(80 \mathrm{mg} \cdot \mathrm{L}^{-1}\right)$ and 530 soluble (Figure S5C). Once purified, we incubated the fusion 531 protein at $37{ }^{\circ} \mathrm{C}$ for four days and assessed whether it binds to $532 \mathrm{Th}-\mathrm{T}$ and CR. Again, the increase in Th-T fluorescence 533 emission (Figure 5A) and the red shift of the CR absorbance 534 (Figure 5B) were indicative of an amyloid-like structure. The 535 band at $1620 \mathrm{~cm}^{-1}$ in the deconvoluted FTIR absorbance 536 spectra confirmed the presence of the intermolecular $\beta$-sheet 537 structure (Figure 5E and Table S1). Finally, TEM images 538 showed that Sup35-5aa-DHFR-Z self-assembled into spherical 539 particles (Figure 5D), whereas the soluble, nonincubated, 540 tandem fusion protein did not form any assembly (Figure 5C). 541 The size of such oligomers, as measured by TEM, was $43.6 \pm$ $5422.8 \mathrm{~nm}$, in good agreement with the $40.1 \pm 5.8 \mathrm{~nm}$ measured 543 by DLS. Higher-magnification TEM images showed the high 544 homogeneity and regular round shape of these nanoparticles 545 (Figure S6). The size of these nanostructures is $\sim 1 / 3$ of that 546 formed by Sup35-5aa-DHFR. This reduction in size can be 547 univocally attributed to the presence of the Z-domain. Sup35-
5aa-DHFR-Z is $\sim 6 \mathrm{kDa}$ larger than Sup35-5aa-DHFR; thus, if 548 we assume that the two nanoparticles display similar 549 compactness, this necessarily implies that the number of 550 molecules in Sup35-5aa-DHFR-Z particles is significantly 551 smaller. This reduction in size upon increasing the dimension 552 of the adjacent domain was already predicted by the above- 553 described relationship between $\mathrm{AR}$ and adjacent globular 554 domains. ${ }^{15}$ The larger the molecule appended to the AR, the 555 lower the number of units that can be incorporated into the 556 amyloid-like oligomers, and the smaller the size of the resulting 557 particle.

Sup35-5aa-DHFR-Z Bifunctional Nanoparticles are 559 Biocompatible. One of the main limitations of using 560 amyloid-like materials in biomedical applications is that they 561 might possess cytotoxic activity. ${ }^{29}$ To discard this possibility, 562 we tested the cytotoxicity of the Sup35-5aa-DHFR-Z nano- 563 particles at different concentrations, ranging from 0.5 to 60564 $\mu \mathrm{M}$, using the PrestoBlue assay (Figure 5F). The statistical 565 analysis using a one-way ANOVA test indicated that the 566 particles did not exhibit significant toxicity for human HeLa 567 cells, suggesting that they would have excellent biocompati- 568 bility.

Dual-Binding Activity of Sup35-5aa-DHFR-Z Nano- 570 particles. To confirm that both DHFR and the Z-domain 571 keep their native structure and functionality in Sup35-5aa- 572 
A

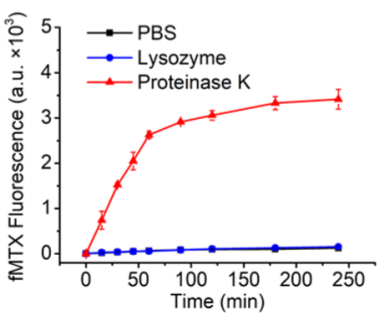

C
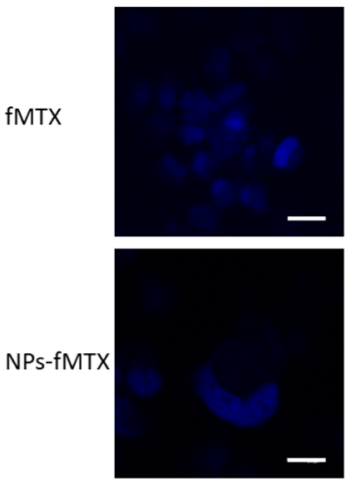

B

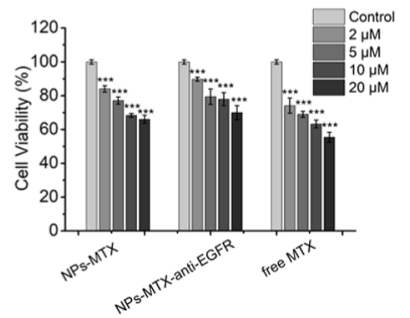

CellMask
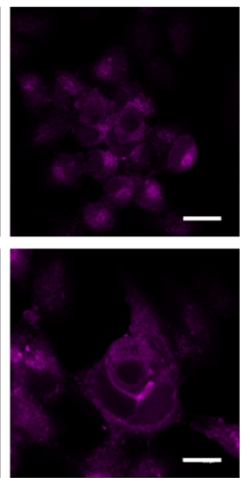

fMTX
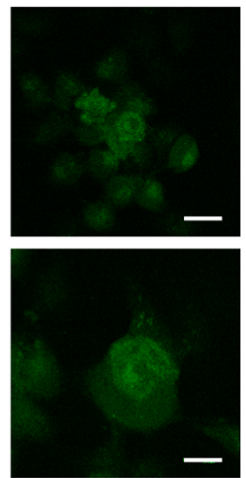

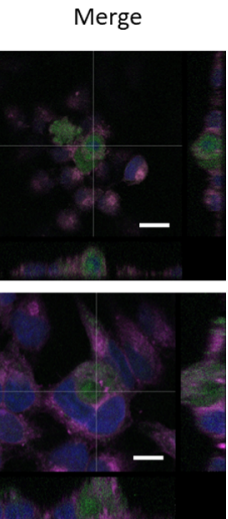

Figure 8. Release, uptake, and cytotoxicity of MTX in Sup35-5aa-DHFR-Z nanoparticles. (A) In vitro release of fluorescein-labeled MTX from Sup35-5aa-DHFR-Z nanoparticles in PBS buffer in the presence of $1 \mu \mathrm{g} \cdot \mathrm{mL}^{-1}$ proteinase $\mathrm{K}$ (red line) at $37^{\circ} \mathrm{C}(n=2)$. The fluorescence emission of fMTX at $515 \mathrm{~nm}$ in the supernatant was measured at the indicated time points. Nanoparticles treated with PBS (black line) and lysozyme (blue line) were used as negative controls. (B) Viability of HeLa cells in the presence of free MTX, MTX-loaded nanoparticles (NPs-MTX), or MTXand anti-EGFR-loaded nanoparticles (NPs-MTX-anti-EGFR). PBS buffer was used as a control. A final concentration of $5 \mathrm{ng} \cdot \mathrm{mL}^{-1} \mathrm{proteinase} \mathrm{K}$ was added to the medium to mimic the proteinase-enriched microenvironment of tumors. Results are expressed as means \pm SD $(n=3)$ and analyzed using a one-way ANOVA test. The statistical differences between the control group and the test group were established at $P<0.001$. (C) Representative confocal images of HeLa cells incubated with free fMTX or fMTX-loaded nanoparticles (NPs-fMTX) preincubated with proteinase K. Nuclei were stained with DAPI, and membranes were stained with CellMask Deep Red. Fluorescein-labeled MTX (fMTX) was detected in the FITC channel. Scale bar represents $10 \mu \mathrm{m}$.

573 DHFR-Z nanoparticles, we incubated them with fMTX (green 574 fluorescence) and an anti-EGFR antibody labeled with Alexa 575 fluor 555 (red fluorescence). Then, they were precipitated and 576 washed three times to remove any unbound molecule and 577 resuspended in PBS buffer. When imaged using fluorescence 578 microscopy, the particles appeared green and red in the 579 respective channels, and the two signals overlapped when the 580 channels were merged (Figure 6). This indicated that the $581 \mathrm{DHFR}$ and the Z-domain embedded in the spherical 582 nanoparticles were bound to fMTX and the antibody, 583 respectively. In contrast, the fibrils formed by the SAC584 Sup35 peptide alone did not bind any of the two molecules, 585 confirming that the observed binding to Sup35-5aa-DHFR-Z 586 nanoparticles does not result from an unspecific interaction of 587 the reagents with the common amyloid-like structure.

\section{Sup35-5aa-DHFR-Z-Functionalized Nanoparticles} 589 Target Cancer Cells Specifically. We assessed if Sup35590 5aa-DHFR-Z nanoparticles can target specific antigens in living 591 cells once they have been loaded with antibodies through their 592 multiple Z-domains. Nanoparticles loaded with red-labeled 593 anti-EGFR antibody (NPs-anti-EGFR) were incubated with $594 \mathrm{HeLa}$ cells, which are known to overexpress EFGR at their 595 membranes. ${ }^{30}$ As can be observed in Figure $7 \mathrm{~A}$, confocal 596 microscopy fluorescence images indicated that the vast 597 majority of the cells were red fluorescent and, therefore, that 598 the multivalent NPs-anti-EGFR had recognized them. In 599 contrast, when the Sup35-5aa-DHFR-Z nanoparticles were 600 loaded with a green-labeled anti-CD3 antibody and incubated with HeLa cells, no cellular labeling was detected, consistent 601 with the fact that this cell type does not express the CD3 602 complex. Thus, the data indicated that the recognition of HeLa 603 cells by NPs-anti-EGFR was antibody-driven and specific. 604

Next, we loaded Sup35-5aa-DHFR-Z nanoparticles, with 605 both fMTX and anti-EGFR antibody (NPs-fMTX-anti-EGFR), 606 with fMTX and a secondary goat antirabbit antibody (NPs- 607 fMTX-antirabbit), or only with fMTX (NPs-fMTX), and we 608 added the three functionalized protein nanoparticles to HeLa 609 cell cultures. After washing the cells with PBS, they were 610 immediately analyzed by flow cytometry, monitoring the green 611 fluorescence of fMTX, present in all three nanoparticles, with 612 an FITC emission detector. Only HeLa cells treated with NPs- 613 fMTX-Anti-EGFR exhibited fMTX fluorescence (Figure 7B), 614 whereas no significant fluorescence was detected for HeLa cells 615 treated with NPs-fMTX-antirabbit or NPs-fMTX. Flow 616 cytometry is quantitative, and the analysis indicated that 617 $>70 \%$ of the cells were bound to NPs-fMTX-anti-EGFR, which 618 shows a high binding affinity of the functionalized nano- 619 particles for EGFR expressing cells. Indeed, confocal images of 620 cells incubated with NPs-fMTX-anti-EGFR indicated that they 621 target the cell membrane (Figure S7).

Overall, it appears that Sup35-5aa-DHFR-Z nanoparticles 623 provide a modular strategy to target any cell type of interest, 624 just by incorporating the adequate antibody thanks to their 625 strong interaction with the properly folded Z-domains and the 626 multivalency of the assembly. 
628 Proteinase-Controlled Release of MTX from Sup35629 5aa-DHFR-Z Nanoparticles. Cancer cells secrete a signifi630 cant number of proteinases, including metalloproteinases, ${ }^{31}$ 631 serine proteinases, ${ }^{32}$ and cathepsins. ${ }^{33}$ This feature has been 632 exploited to develop proteinase-responsive nanocarriers ${ }^{34}$ and 633 polymer nanoshells. ${ }^{35}$

634 It is known that amyloid fibrils are highly resistant to 635 proteolysis, but we hypothesized that the globular domains 636 hanging from the amyloid core in Sup35-5aa-DHFR-Z 637 nanoparticles would be protease-sensitive. This will open an 638 opportunity to use the DHFR moiety as a carrier for MTX 639 under the assumption that the inhibitor will be released from 640 the oligomers once they arrive at a protease-rich environment 641 and the accessible DHFR domains would be proteolytically 642 attacked.

643 We incubated fMTX-loaded nanoparticles with the broad644 spectrum proteinase $\mathrm{K}(\mathrm{PK})$ at a final concentration of $1 \mu \mathrm{g}$. $645 \mathrm{~mL}^{-1}$ and measured the kinetics of fluorescein fluorescence 646 apparition in the supernatant, upon centrifugation (Figure 8A). 647 The nanoparticles were incubated also in PBS alone or treated 648 with lysozyme at the same concentration to ensure that any 649 observed release is not spontaneous or unspecific. In contrast 650 to control samples, PK-treated nanoparticles showed a 651 continuous delivery of fMTX to the solution, with a cumulative 652 release efficiency $>90 \%$ after 4 h of reaction.

653 An SDS-PAGE of the nanoparticles indicated that they were 654 SDS-sensitive since, despite high molecular species being 655 observed, the vast majority of the fusion protein ran as a 656 monomer. This sensitivity to the detergent is typical of 657 amyloid oligomers. ${ }^{36}$ This allowed us to follow the PK 658 digestion by SDS-PAGE, which confirmed that the nano659 particles are proteinase-sensitive and that no intact Sup35-5aa660 DHFR-Z fusion remains after $45 \mathrm{~min}$ and neither does any 661 fragment compatible with an intact DHFR domain (Figure 662 S8), explaining why fMTX is released from the protein 663 nanoparticles.

664 Internalization of MTX Released from Loaded Nano665 particles. We investigated if the DHFR inhibitor released 666 from NPs-fMTX can be uptaken by HeLa cells. To this aim, we 667 incubated the cells with $10 \mu \mathrm{M}$ free fMTX or fMTX-loaded 668 nanoparticles, both preincubated with $50 \mathrm{ng} \cdot \mathrm{mL}^{-1} \mathrm{PK}$ for 5 $669 \mathrm{~min}$, followed by protease inactivation with EDTA. After $4 \mathrm{~h}$ of 670 incubation, the medium was removed, and the cells were 671 washed with the medium three times. Then, cells were stained 672 with DAPI and CellMask Deep Red for nuclei and membrane 673 visualization, respectively. Intracellular green fluorescent fMTX 674 was observed by confocal microscopy for both free added 675 fMTX and NPs-fMTX (Figure 8C), indicating that the 676 nanoparticle-released inhibitor is efficiently internalized.

677 MTX-Loaded Nanoparticles Induce Death of Cancer 678 Cells. We have demonstrated that the MTX-loaded nano679 particles can liberate DHFR-bound MTX and that the 680 inhibitor can internalize into cancer cells. This immediately 681 suggested that MTX-loaded nanoparticles could induce 682 proteolysis-mediated cell death. Therefore, we measured the 683 viability of HeLa cells in the presence of NPs-MTX, NPs684 MTX-anti-EGFR, or free MTX. A final concentration of $5 \mathrm{ng}$. $685 \mathrm{~mL}^{-1} \mathrm{PK}$ was added to the cell culture medium to mimic the 686 proteinase-enriched microenvironment of tumor tissues. In all 687 cases, a significant dose-dependent decrease in HeLa cell 688 viability was observed, compared with control cells incubated 689 with PK only (Figure 8B). The concentration of proteinase in 690 the assay is lower than the one described in the tumors' extracellular environment; ${ }^{37}$ still, it is sufficient to promote 691 MTX release from the nanoparticles, internalization of the 692 inhibitor, and cancer cell death. Importantly, untreated and 693 PK-treated Sup35-5aa-DHFR-Z nanoparticles are not toxic for 694 nontumoral lung MRC-5 cells (Figure S9), indicating that the 695 multifunctional nanoparticles might be safe for normal cells in 696 vivo.

\section{CONCLUSIONS}

In the present work, we first demonstrated that by modulating 699 interdomain linker length, one could attain a tight control of 700

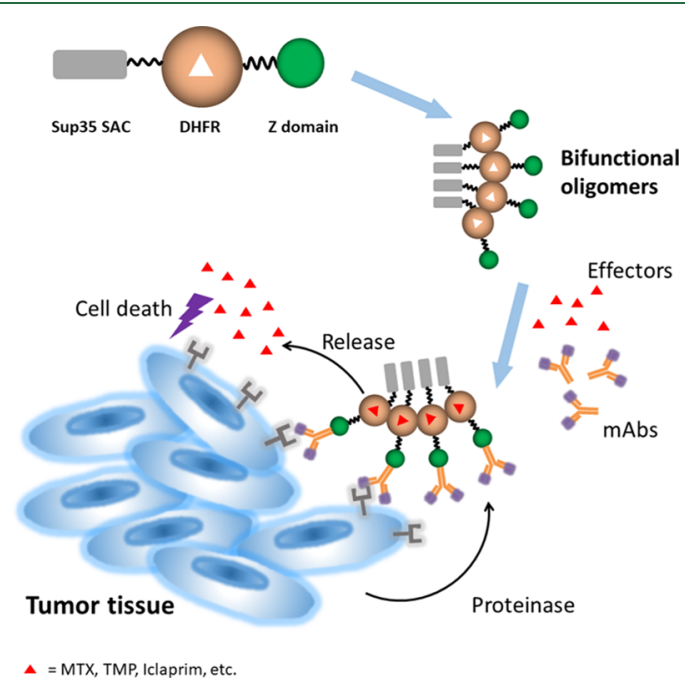

Figure 9. Schematic illustration of bifunctional amyloid oligomeric nanoparticles and their potential application for targeted drug delivery. The construct consists of the Sup35 soft amyloid core (Sup35-SAC, gray square), dihydrofolate reductase (DHFR, orange circle), and the Z-domain derived from protein A (green circle). The short linker between Sup35-SAC and DHFR enables the fusion protein to self-assemble into stable bifunctional oligomeric nanoparticles. Drugs (red triangles) and monoclonal antibodies (mAbs) loaded into the nanoparticles would target the tumor cells and release the drugs in a proteinase-enriched environment. As a consequence, the released drugs are internalized and induce the death of the targeted tumor cells.

the mesoscopic properties of the resulting amyloid-like 701 nanostructures. This strategy does not require intricate 702 structural engineering since it relies on fundamental bio- 703 physical principles. The approach allowed us to generate 704 oligomeric amyloid-like nanoparticles. These spherical nano- 705 particles are homogenous in size, stable, and biocompatible. 706 Besides, the multiple globular domains they contain are highly 707 active in the assembled state, as shown here for DHFR and the 708 Z-domain. Therefore, they constitute a novel kind of functional 709 and functionalizable nanomaterial.

710

We provide a proof-of-concept of the utility of these de 711 novo-designed nanostructures by showing how they can be 712 decorated with an antibody of interest, which act as an 713 antenna, directing the multivalent nanoparticles to the specific 714 cell types expressing the selected antigen at their surfaces, 715 allowing the discrimination between diseased and functional 716 cells. When this ability is combined with the capability of 717 DHFR to carry and shield MTX, avoiding nonspecific toxic 718 effects, it appears as a very appealing strategy for targeted 719 delivery of the drug in the proteinase-enriched microenviron- 720 ment of tumors, which is followed by MTX internalization into $721 \mathrm{f} 9$ 
722 the cytosol and localized killing of cancer cells (Figure 9). 723 Overall, the multifunctional, self-assembled amyloid nano724 particles we present here constitute a new and safe nano725 technological modular scaffold with the potential of facilitating 726 the specific delivery of agents to specific sites in the body, 727 overpassing the major barrier for bioimaging and tissue728 targeted therapies. Importantly, the building blocks of these 729 nanostructures can be produced at high yield and purified at 730 homogeneity from the soluble cell fraction, which results in 731 reduced costs when compared with alternative nanomedicines.

\section{$732 \square$ ASSOCIATED CONTENT}

\section{SI Supporting Information}

734 The Supporting Information is available free of charge at 735 https://pubs.acs.org/doi/10.1021/acs.biomac.0c01103.

736 Characterization of fusion proteins; FTIR spectrum of 737 DHFR-wt; DLS size distribution of the different 738 assemblies; high-magnification TEM image of Sup35739 5aa-DHFR-Z nanoparticles; confocal microscopy images 740 of HeLa cells incubated with nanoparticles; SDS-PAGE 741 analysis of proteinase $\mathrm{K}$ digestion; viability of MRC-5 742 cells; assignment of secondary structure components in 743 the FTIR spectra; kinetics and thermodynamic dissoci744 ation constants of the assemblies (PDF)

\section{$745 \square$ AUTHOR INFORMATION}

\section{Corresponding Author}

747 Salvador Ventura - Institut de Biotecnologia i Biomedicina and 748 Departament de Bioquimica i Biologia Molecular, Universitat 749 Autònoma de Barcelona, 08193 Bellaterra, Spain;

750 orcid.org/0000-0002-9652-6351; Phone: 34-93-5868956;

751 Email: salvador.ventura@uab.es; Fax: 34-93-5811264

\section{Authors}

753 Weiqiang Wang - Institut de Biotecnologia i Biomedicina and

754 Departament de Bioquimica i Biologia Molecular, Universitat

755 Autònoma de Barcelona, 08193 Bellaterra, Spain;

756 orcid.org/0000-0002-6962-8811

757 Rafayel A. Azizyan - Centre de Recherche en Biologie cellulaire 758 de Montpellier, UMR 5237 CNRS, Universite Montpellier,

759 Montpellier 34090, France; Institut de Biologie

760 Computationnelle, Université Montpellier, Montpellier 34090,

\section{$761 \quad$ France}

762 Adriana Garro - Universidad Nacional de San Luis IMASL-

763 CONICET, San Luis D5702, Argentina

764 Andrey V. Kajava - Centre de Recherche en Biologie cellulaire de

765 Montpellier, UMR 5237 CNRS, Universite Montpellier,

766 Montpellier 34090, France; Institut de Biologie

767 Computationnelle, Université Montpellier, Montpellier 34090,

768 France; (1) orcid.org/0000-0002-2342-6886

769 Complete contact information is available at:

770 https://pubs.acs.org/10.1021/acs.biomac.0c01103

\section{Notes}

772 The authors declare no competing financial interest.

\section{ACKNOWLEDGMENTS}

774 We acknowledge the technical support from the Cell Culture 775 Unit of Servei de Cultius Cel-lulars i Producció d'Anticossos i 776 Citometria (SCAC) and from the Servei de Microscopia of 777 Universitat Autònoma de Barcelona (UAB). This work was 778 funded by the Spanish Ministry of Economy and Competitive- ness BIO2016-78310-R to S.V and by ICREA, ICREA- 779 Academia 2015, to S.V. W.W. acknowledges financial support 780 from the China Scholarship Council (CSC): No. 781 201606500007.

\section{REFERENCES}

783

(1) Knowles, T. P.; Mezzenga, R. Amyloid Fibrils as Building Blocks 784 for Natural and Artificial Functional Materials. Adv. Mater. 2016, 28, 785 6546-6561.

(2) Ross, C. A.; Poirier, M. A. Protein Aggregation and 787 Neurodegenerative Disease. Nat. Med. 2004, 10, S10-S17. 788

(3) Fowler, D. M.; Koulov, A. V.; Balch, W. E.; Kelly, J. W. 789 Functional Amyloid-from Bacteria to Humans. Trends Biochem. Sci. 790 2007, 32, 217-224.

(4) Wei, G.; Su, Z.; Reynolds, N. P.; Arosio, P.; Hamley, I. W.; Gazit, 792 E.; Mezzenga, R. Self-Assembling Peptide and Protein Amyloids: from 793 Structure to Tailored Function in Nanotechnology. Chem. Soc. Rev. 794 2017, 46, 4661-4708.

(5) Rufo, C. M.; Moroz, Y. S.; Moroz, O. V.; Stöhr, J.; Smith, T. A.; 796 Hu, X.; DeGrado, W. F.; Korendovych, I. V. Short Peptides Self- 797 Assemble to Produce Catalytic Amyloids. Nat. Chem. 2014, 6, 303- 798 309.

(6) Chiti, F.; Dobson, C. M. Protein Misfolding, Amyloid 800 Formation, and Human Disease: A Summary of Progress Over the 801 Last Decade. Annu. Rev. Biochem. 2017, 86, 27-68. 802

(7) Wang, W.; Navarro, S.; Azizyan, R. A.; Baño-Polo, M.; 803 Esperante, S. A.; Kajava, A. V.; Ventura, S. Prion Soft Amyloid 804 Core Driven Self-Assembly of Globular Proteins into Bioactive 805 Nanofibrils. Nanoscale 2019, 11, 12680-12694.

(8) Sant'Anna, R.; Fernández, M. R.; Batlle, C.; Navarro, S.; De 807 Groot, N. S.; Serpell, L.; Ventura, S. Characterization of Amyloid 808 Cores in Prion Domains. Sci. Rep. 2016, 6, No. 34274.

(9) Sabate, R.; Rousseau, F.; Schymkowitz, J.; Ventura, S. What 810 Makes a Protein Sequence a Prion? PLoS Comput. Biol. 2015, 11, 811 No. e1004013.

(10) Sabate, R.; Rousseau, F.; Schymkowitz, J.; Batlle, C.; Ventura, S. 813 Amyloids or Prions? That is the Question. Prion 2015, 9, 200-206. 814 (11) Wasmer, C.; Schütz, A.; Loquet, A.; Buhtz, C.; Greenwald, J.; 815 Riek, R.; Böckmann, A.; Meier, B. H. The Molecular Organization of 816 the Fungal Prion HET-s in its Amyloid Form. J. Mol. Biol. 2009, 394, 817 119-127.

818

(12) Baxa, U.; Cassese, T.; Kajava, A. V.; Steven, A. C. Structure, 819 Function, and Amyloidogenesis of Fungal Prions: Filament Poly- 820 morphism and Prion Variants. Adv. Protein Chem. 2006, 73, 125-180. 821

(13) Li, J.; McQuade, T.; Siemer, A. B.; Napetschnig, J.; Moriwaki, 822 K.; Hsiao, Y.-S.; Damko, E.; Moquin, D.; Walz, T.; McDermott, A.; 823 et al. The RIP1/RIP3 Necrosome Forms a Functional Amyloid 824 Signaling Complex Required for Programmed Necrosis. Cell 2012, 825 $150,339-350$.

(14) Chen, A. K.-H.; Lin, R. Y.-Y.; Hsieh, E. Z.-J.; Tu, P.-H.; Chen, 827 R. P.-Y.; Liao, T.-Y.; Chen, W.; Wang, C.-H.; Huang, J. J.-T. Induction 828 of Amyloid Fibrils by the C-terminal Fragments of TDP-43 in 829 Amyotrophic Lateral Sclerosis. J. Am. Chem. Soc. 2010, 132, 1186- 830 1187.

(15) Azizyan, R. A.; Garro, A.; Radkova, Z.; Anikeenko, A.; Bakulina, 832 A.; Dumas, C.; Kajava, A. V. Establishment of Constraints on Amyloid 833 Formation Imposed by Steric Exclusion of Globular Domains. J. Mol. 834 Biol. 2018, 430, 3835-3846.

835

(16) Kajava, A. V.; Baxa, U.; Wickner, R. B.; Steven, A. C. A Model 836 for Ure2p Prion Filaments and Other Amyloids: The Parallel 837 Superpleated $\beta$-Structure. Proc. Natl. Acad. Sci. U.S.A. 2004, 101, 838 $7885-7890$.

(17) Azizyan, R. A.; Wang, W.; Anikeenko, A.; Radkova, Z.; 840 Bakulina, A.; Garro, A.; Charlier, L.; Dumas, C.; Ventura, S.; Kajava, 841 A. V. Amyloidogenicity as a Driving Force for the Formation of 842 Functional Oligomers. J. Struct. Biol. 2020, 212, No. 107604. 
844 (18) Schnell, J. R.; Dyson, H. J.; Wright, P. E. Structure, Dynamics, 845 and Catalytic Function of Dihydrofolate Reductase. Annu. Rev. 846 Biophys. Biomol. Struct. 2004, 33, 119-140.

847 (19) Tashiro, M.; Tejero, R.; Zimmerman, D. E.; Celda, B.; Nilsson, 848 B.; Montelione, G. T. High-Resolution Solution NMR Structure of 849 the Z Domain of Staphylococcal Protein A. J. Mol. Biol. 1997, 272, $850573-590$.

851 (20) Forsgren, A.; Sjöquist, J. "Protein A" from S. aureus: I. Pseudo852 immune Reaction with Human $\gamma$-globulin. J. Immunol. 1966, 97, 822853827.

854 (21) Huffman, D. H.; Wan, S. H.; Azarnoff, D. L.; Hoogstraten, B. 855 Pharmacokinetics of Methotrexate. Clin. Pharmacol. Ther. 1973, 14, $856572-579$.

857 (22) Blaney, J. M.; Hansch, C.; Silipo, C.; Vittoria, A. Structure858 Activity Relationships of Dihydrofolated Reductase Inhibitors. Chem. 859 Rev. 1984, 84, 333-407.

860 (23) Qindeel, M.; Khan, D.; Ahmed, N.; Khan, S.; Rehman, A. U. 861 Surfactant-Free, Self-Assembled Nanomicelles-Based Transdermal 862 Hydrogel for Safe and Targeted Delivery of Methotrexate against 863 Rheumatoid Arthritis. ACS Nano 2020, 14, 4662-4681.

864 (24) Sadrjavadi, K.; Shahbazi, B.; Fattahi, A. De-Esterified 865 Tragacanth-Chitosan Nano-Hydrogel for Methotrexate Delivery; 866 Optimization of the Formulation by Taguchi Design. Artif. Cells, 867 Nanomed., Biotechnol. 2018, 46, 883-893.

868 (25) Tawfik, M. K. Combination of Coenzyme Q10 with 869 Methotrexate Suppresses Freund's Complete Adjuvant-Induced 870 Synovial Inflammation with Reduced Hepatotoxicity in Rats: Effect 871 on Oxidative Stress and Inflammation. Int. Immunopharmacol. 2015, 872 24, 80-87.

873 (26) Choi, G.; Kim, T.-H.; Oh, J.-M.; Choy, J.-H. Emerging 874 Nanomaterials with Advanced Drug Delivery Functions; Focused on 875 Methotrexate Delivery. Coord. Chem. Rev. 2018, 359, 32-51.

876 (27) Bystroff, C.; Oatley, S. J.; Kraut, J. Crystal structures of 877 Escherichia coli dihydrofolate reductase: The NADP + holoenzyme 878 and the Folate. cntdot. NADP+ Ternary Complex. Substrate Binding 879 and a Model for the Transition State. Biochemistry 1990, 29, 32638803277.

881 (28) Moks, T.; ABRAHMSÉN, L.; NILSSON, B.; HELLMAN, U.; 882 SJÖQUIST, J.; UHLÉN, M. Staphylococcal Protein A Consists of 883 Five IgG-Binding Domains. Eur. J. Biochem. 1986, 156, 637-643.

884 (29) Díaz-Caballero, M.; Navarro, S.; Ventura, S. Soluble Assemblies 885 in the Fibrillation Pathway of Prion-Inspired Artificial Functional 886 Amyloids are Highly Cytotoxic. Biomacromolecules 2020, 21, 23348872345

888 (30) Laskin, J. J.; Sandler, A. B. Epidermal Growth Factor Receptor: 889 A Promising Target in Solid Tumours. Cancer Treat. Rev. 2004, 30, $890 \quad 1-17$.

891 (31) Folgueras, A. R.; Pendas, A. M.; Sanchez, L. M.; Lopez-Otin, C. 892 Matrix Metalloproteinases in Cancer: From New Functions to 893 Improved Inhibition Strategies. Int. J. Dev. Biol. 2004, 48, 411-424. 894 (32) Koshikawa, N.; Yasumitsu, H.; Umeda, M.; Miyazaki, K. 895 Multiple Secretion of Matrix Serine Proteinases by Human Gastric 896 Carcinoma Cell Lines. Cancer Res. 1992, 52, 5046-5053.

897 (33) Koblinski, J. E.; Dosescu, J.; Sameni, M.; Moin, K.; Clark, K.; 898 Sloane, B. F. Interaction of Human Breast Fibroblasts with Collagen I 899 Increases Secretion of Procathepsin B. J. Biol. Chem. 2002, 277, $90032220-32227$.

901 (34) Zhu, L.; Kate, P.; Torchilin, V. P. Matrix Metalloprotease 2902 Responsive Multifunctional Liposomal Nanocarrier for Enhanced 903 Tumor Targeting. ACS Nano 2012, 6, 3491-3498.

904 (35) Yang, J.; Yang, Y.; Kawazoe, N.; Chen, G. Encapsulation of 905 Individual Living Cells with Enzyme Responsive Polymer Nanoshell. 906 Biomaterials 2019, 197, 317-326.

907 (36) Gadad, B. S.; Britton, G. B.; Rao, K. Targeting Oligomers in 908 Neurodegenerative Disorders: Lessons from $\alpha$-Synuclein, Tau, and 909 Amyloid- $\beta$ Peptide. J. Alzheimer's Dis. 2011, 24, 223-232.

910 (37) Schmalfeldt, B.; Prechtel, D.; Härting, K.; Späthe, K.; Rutke, S.; 911 Konik, E.; Fridman, R.; Berger, U.; Schmitt, M.; Kuhn, W. Increased 912 Expression of Matrix Metalloproteinases (MMP)-2, MMP-9, and the
Urokinase-type Plasminogen Activator is Associated with Progression 913 from Benign to Advanced Ovarian Cancer. Clin. Cancer. Res. 2001, 7, 914 2396-2404.

\section{5}

